Cahiers $d u$ MONDE RUSSE

\section{Cahiers du monde russe}

Russie - Empire russe - Union soviétique et États indépendants

$61 / 3-4 \mid 2020$

Écritures visuelles, sonores et textuelles de la justice

\title{
La Roue rouge : le chaos vaincu par l'écriture?
}

\section{Georges Nivat}

\section{OpenEdition \\ Journals}

Édition électronique

URL : https://journals.openedition.org/monderusse/12264

DOI : 10.4000/monderusse. 12264

ISSN : $1777-5388$

Éditeur

Éditions de l'EHESS

Édition imprimée

Date de publication : 1 juillet 2020

Pagination : 563-568

ISBN : 978-2-7132-2832-2

ISSN : $1252-6576$

Référence électronique

Georges Nivat, "La Roue rouge : le chaos vaincu par l'écriture ? ", Cahiers du monde russe [En ligne] 61/3-4 | 2020, mis en ligne le 01 juillet 2020, consulté le 04 septembre 2022. URL : http://

journals.openedition.org/monderusse/12264; DOI : https://doi.org/10.4000/monderusse.12264

Tous droits réservés 
Richard TEMPEST

Overwriting Chaos

Aleksandr Solzhenitsyn's Fictive Worlds

Boston : Academic Studies Press, 2019, 750 p.

\section{La Roue rouge : le chaos vaincu par l'écriture?}

Ce n'est qu'à la fin de son ouvrage que Richard Tempest explique et résume sa conception de La Roue rouge : « un gigantesque collage de textes et de topos $»^{1}$ unifié par les personnages de fiction qui servent d' « adhésif humain $»^{2}$ pour coller ensemble une « dégringolade » d'envergure mondiale. Cette dégringolade, c'est la révolution russe. En d'autres mots, Tempest veut nous rendre attentifs à l'usage de plus en plus dense de procédés narratifs modernistes, autrement dit destructifs dans le grand texte historien de Solženicyn. S'il a raison, c'est que Solženicyn recourt en quelque sorte à l'armement de son adversaire, car il ne fait pas de doute que le modernisme est son adversaire.

«Le pouvoir soviétique a trouvé son Homère en Solženicyn $»^{3}$ : cette affirmation, que nous ne contestons pas, est plutôt paradoxale chez Tempest. Car Homère n'entre pas dans les catégories ni du modernisme ni du réalisme passéiste. L'ambition de Tempest est haute, mais il n'est pas le premier ; on pourrait lui reprocher de ne tenir quasiment aucun compte des ouvrages qui l'ont précédé, et il est vrai que les États-Unis ont toujours été en retard dans la traduction comme dans l'exégèse de Solženicyn. Ni Strada, ni Olivier Clément, pour donner deux exemples, ne sont vraiment retenus. Certes, l'envergure de l'entreprise est impressionnante, et Tempest tient presque à bout de bras l'ensemble de l'œuvre, ce qui n'est pas facile.

Solženicyn, s'il n'est pas le Homère du régime qui a nom soviétique, peut en effet être dit le Homère du temps terrible que cet ordre a imposé à trois générations d'êtres humains, y compris les écrivains, réduits souvent à l'état de laquais, ou de victimes sanguinolentes (songeons à Babel, à Meyerhold, à Mandelstam, et tant d'autres dans ce martyrologe). Certains ont toutefois surmonté par leur écriture la terreur, la peur permanente qui collait à tous, et surtout clairement vu le despotisme arriver sur le monde. « Soviétiques » furent Mandelstam, Ahmatova, Pasternak, en ce sens que, sans ce régime de loup, ils n'auraient pas été ce qu'ils furent. Sans la nuit soviétique, leur lucidité ne nous aurait pas éclairés comme celles des grands prophètes juifs.

En dépit de son sous-titre, Tempest a été contraint de tenir compte des œuvres de non-fiction. Chez un écrivain formé par la guerre, la prison et le camp de travail forcé l'ont contraint d'abandonner son marxisme romantique, de se dessiller les yeux et, finalement, d'entamer une lutte, un corps-à-corps avec la tyrannie. Aurait-il été un Homère sans la révolution, la trahison de la révolution, l'esclavage enfanté par la révolution? Qui pourrait le dire...?

Tempest divise son ouvrage en deux grandes parties, «In situ » et « Ex situ », autrement dit En Russie et Hors de Russie, soulignant le rôle de l'exil dans cette 
œuvre. Un mot d'abord pour rappeler que cet exil est celui d'un proscrit, comme pour Hugo, et ceux du « bateau des philosophes » en 1922. Pas une fuite, comme ce fut très souvent le cas (relisons Bulgakov), même si cette fuite était amplement justifiée (relisons Šmelëv). Tempest raconte la « non-rencontre » à Montreux entre Nabokov et Solženicyn, et analyse l'influence possible de l'un sur l'autre. L'auteur de Bend Sinister était évidemment un de ceux qui, avec lucidité, avaient vaincu le chaos, mais il n'était pas un proscrit. Et Nabokov, en somme, eut une vie heureuse, ex situ était pour lui devenu in situ, l'exil était devenu sa maison.

Tel ne fut pas le cas pour Solženicyn. Après les contraintes les plus sévères (guerre, prison, cancer, écriture clandestine), ce fut l'exil américain - une écriture longue de dix-sept-ans de vie, une organisation quasi monacale de la vie quotidienne. Autrement dit, après la contrainte du dehors, la contrainte du dedans. L'une nous a donné L'Archipel du goulag, l'autre nous a donné La Roue rouge, un roman historique de plus de six mille pages. Ce diptyque, dont Tempest fait l'axe de son livre, correspond-il à un vrai, profond tournant de l'œuvre? Conrad, Nabokov, (on peut rajouter Romain Gary ${ }^{4}$, non moins génial) ont changé de langue, pas Solženicyn. Il emporta la langue avec lui, comme il emportait le dictionnaire Dahl, qui l'a suivi partout. Un autre diptyque élaboré par Tempest fait appel à Roland Barthes et son distinguo entre récits fonctionnels (les contes) et récits indiciels (les romans psychologiques), les premiers informant sur le « faire » des personnages, et les seconds sur leur « être ». Solženicyn va du fonctionnel à l'indiciel, autrement dit d'un clacissisme de $\mathrm{XIX}^{\mathrm{e}}$ siècle à un modernisme de $\mathrm{XX}^{\mathrm{e}}$ siècle. Un modernisme, rappelons-le, pourtant voué aux gémonies par Solženicyn dans ses textes de nonfiction. C'est une des idées essentielles du livre ; elle n'est pas entièrement neuve, mais Tempest en fait son axe.

D'autres types de diptyques avaient déjà été étudiés sous d'autres aspects : par exemple celui du chiasme entre le temps de l'écriture et le temps de la narration. Du premier point de vue, L'Archipel vient en premier, La Roue en second; du second point de vue, c'est l'inverse. La Roue nous dit l'enchaînement qui amène à la création de l'enfer, autrement dit à L'Archipel du goulag, c'est la chronologie causale (la fable selon Šklovskij). Mais L'Archipel précédant La Roue, c'est le vrai texte soljenitsynien, le sujet, au sens chklovskien. Cette structure de l'œuvre de Solženicyn a été étudiée par le critique russe Andrej Nemzer ${ }^{5}$ dans un livre qui a fait date dans les études soljénitsyniennes. Autre diptyque, moi-même ai à ce sujet apporté la comparaison avec Jean-Paul Sartre (une autre «non-rencontre »), car Sartre était orphelin de père comme Solženicyn, et il commence ce qui est à mes yeux son meilleur livre, Les Mots, par une comparaison de lui avec Énée portant Anchise sur ses épaules. Le père devient le fils de son fils. C'est ce qui arrive à San'ja Laženicyn et aussi à l'autre alter ego de Solženicyn, Neržin. Pour en revenir au diptyque fondamental adopté par Tempest, In situ / Ex situ, il me semble que ce déplacement d'un continent à l'autre, dont Iosif Brodskij a fait l'axe de son œuvre, n'est pas chez Solženicyn essentiel. Mais il structure ce livre.

Le destinataire du livre n'est pas clairement défini. Est-ce « l'honnête homme », comme on dit en français, sont-ce les collègues américains, ou encore l'étroit cercle 
international des spécialistes ? Un jargon narratologique à la Gérard Genette, parfois presque ubuesque, peut rebuter l'honnête homme. Mais une façon directe de s'adresser au lecteur crée une intimité inattendue qu'il ne peut qu'apprécier. Grand amateur de trouvailles linguistiques, Tempest est peut-être son premier destinataire. Il adore déchiffrer la signification des noms et prénoms, un peu comme s'il gardait sous la main, en livre de chevet, la Légende dorée de Jacobus de Voragine. Il juge les personnages un peu comme s'il les rencontrait lui-même ; par exemple, il nous montre le jeune avocat Kerenskij criant par la fenêtre, comme le marquis de Sade d'une embrasure de la Bastille, pour indiquer à la foule qu'il est là, et que c'est lui le plus intéressant. Ou encore il apporte au lecteur le sonnet de Shelley sur « Ozymandias, Roi des Rois », et dont il ne reste qu'un débris de marbre en plein désert. On sent là un réel plaisir d'écriture, vraie rareté en territoire universitaire.

Hélas, une dimension essentielle de l'œuvre me semble absente : la dimension de la confession. Les reconstructions « automythobiographiques », nous dit l'auteur, jouent un grand rôle dans La Roue et d'autres textes encore. Mais l'élément fondateur de cette « automythobiographie », c'est la confession. Certes Solženicyn a sûrement moins péché qu'Augustin (n'ayant eu ni le temps, ni la tentation!), mais la honte et la fureur de cette honte sont assez proches par moments du Père de l'Église. Quant à ce qu'il baptise l'andocentrisme de Solženicyn, certes, prison, armée, bagne sont des lieux d'enfermement d'hommes, privés ou presque de femmes. Les amourettes de Neržin à la šaraška (prison-laboratoire), celles d'Oleg au Pavillon des cancéreux sont d'assez pâles exceptions. Le «masculinisme », éros exclusivement masculin, figure de façon plus convaincante, dans les scènes où le soldat Arsenij Blagodarëv, rentré en permission chez lui, fouette sa femme dans le secret de leur étuve. C'est ce que prescrit le Ménagier russe du XVI ${ }^{\mathrm{e}}$ siècle, et il y a là un côté macho qui n'emprunte rien à la vie de l'auteur, mais tout à une certaine idéologie archaïsante. Quant aux amours du colonel Vorotyncev, auxquelles Tempest accorde de très longues analyses, on peut les juger secondaires, on est loin d'Anna Karenin. Parmi les ouvrages que Tempest ne connaît pas, publiés en français, il y a l'ouvrage du théologien orthodoxe Olivier Clément, inventeur de la théologie $d u$ $\operatorname{corps}^{6}$. Je crois que c'est plutôt par ce biais de la sacralité corporelle que l'on peut appréhender Solženicyn. Tolstoj, le contre-exemple obnubilant de Solženicyn, était d'un autre « masculinisme », Le Père Serge, et bien d'autres récits en sont des révélateurs, mais il est allié à une haine pour la femme (La Sonate à Kreutzer), qui, elle, est étrangère à Solženicyn.

Simone Weil a baptisé L'Iliade poème de la violence; ce n'est pas pour la violence que Solženicyn a aimé la guerre, pas pour voir Hector traîner Patrocle derrière son char. Bien qu'il y ait de la violence brute dans La Roue rouge, symbolisée par cette roue de feu qui dévale, elle ne figure que par moments brefs. Il y a surtout la mort et l'attente de la mort, qui habitent les temps de calme dans toute guerre. Octobre 16 est une longue attente dans l'inaction de l'action militaire, c'està-dire une longue attente de la mort possible, et donc réelle ${ }^{7}$. Guerrier-né, le héros de Solženicyn, le colonel Vorotyncev vit cette inaction et voit l'action lui échapper, et échapper à la Russie. Un échec qui est au centre de cette épopée. 
Parmi les derniers récits de Solženicyn, il y a des récits de guerre, et Tempest leur accorde un sort important, les comparant à l'ouvrage de Norman Mailer, Les Nus et les morts. Véritable "Chanson de Roland de Solženicyn », ajoute-t-il et il a bien raison puisque Roncevaux est l'histoire d'une terrible défaite, où la vengeance, la trahison, la haine des siens l'emportent sur le courage chevaleresque. Tempest définit la technique narrative de ce récit, duquel il est le premier à faire une analyse aussi longue. Comme il a fait appel à Barthes, il fait appel à Julia Kristeva, qui lui apprend à voir dans la technique d'Adlig Schwenkitten une logique à la fois épique et théologique, une foi dans le sens littéral du mot. J'épargnerai ici au lecteur des citations qui seraient trop jargonnantes et que je vais « traduire». Il s'agit, en somme, du tressage de codes archiconnus de l'antique épopée avec des procédés narratifs nouveaux, postmodernistes. La parataxe, l'ellipse, la fragmentation jusqu'au mot isolé, comme chez Majakovskij, les rocades continuelles du sujet parlant, ou encore les « déports diégétiques » entre présent de l'action militaire des vingt-quatre heures composant les vingt-quatre petits chapitres du récit et les flash-back sur les années 1920 ou 1930. Ces derniers sont de minuscules excursus, qui disent la gêne du jeune homme, fils d'un général qui connaîtra une période de disgrâce, lorsqu'il découvre les malheurs entraînés par la dékoulakisation. Tempest s'attarde sur ce dernier récit parce qu'il y voit l'aboutissement de ce qu'il entend démontrer dans son vaste ouvrage. J'ajouterais pour ma part que cette fois-ci Solženicyn ne porte pas son père sur ses épaules comme Anchise, il s'est inséré lui-même, mais avec l'extrême modestie du peintre médiéval qui se représente dans un petit recoin de son retable. Une fois de plus, l'épisode de l'encerclement de son régiment, abandonné par le haut commandement, un épisode qui le hante depuis ses Nuits prussiennes composées par cœur à la šaraška, et qui est de retour.

Ici aussi, c'est peut-être l'appréhension du sacré dont on peut regretter l'absence. Car la guerre a chez Solženicyn quelque chose de sacré et, par exemple, l'enterrement du colonel Kabanov, dans Août 14, tout comme le suicide du général Samsonov qui trompe ses subordonnés pendant leur fuite hors de l'étau allemand, font écho à cet épisode vécu par Solženicyn, qui lui-même fait écho aux combats vécus par son propre père dans ces mêmes forêts prussiennes. Ce sont des « hauts points », auxquels Tempest n'est selon moi, pas assez attentif, précisément parce que la présence du sacré dans cette œuvre, si magnifiquement diagnostiquée par Olivier Clément, ne joue guère de rôle dans sa conception, bien plus esthète. Mais il est certain que cette présence du sacré diminue, quand, dans l'immense enchâ̂nement des quatre Nœuds que l'écrivain nous a laissés (sur les vingt qu'il avait conçus), le lecteur avance vers la catastrophe du second Temps des Troubles de l'histoire de la Russie, c'est-à-dire R-17, autrement dit la révolution de 1917, et l'immense narration soljénitsynienne semble s'enfoncer dans un crépuscule.

Quand il aborde la résonance de l'œuvre, Tempest a un grand panel de textes, de références, de comparaisons qui nous ont beaucoup intéressé, bien qu'il y manque quelques noms essentiels. Solženicyn n'est pas né sans contexte et son contexte n'est pas que le réalisme socialiste. Vassilij Grossman est absent, alors que l'auteur 
de Vie et destin, (certes moins « moderniste » que Solženicyn), semble indispensable dans une réflexion sur cette fabrique de l'inhumain dont la littérature russe nous a donné d'extraordinaires récits : Šalamov, Evgenija Ginzburg, Sinjavskij, Georgij Demidov, et tant d'autres. Tempest évoque les adversaires obstinés de Solženicyn, et il a tout à fait raison de le faire. Mais lorsqu'il met sur le même plan un pamphlétaire mesquin, Bušin, et l'auteur des Singulières Aventures d'Ivan Čonkin, Vladimir Vojnovič, un grand créateur de la résistance par le burlesque, c'est une injustice inexplicable, même si, bien sûr, Vojnovič était un adversaire opiniâtre de Solženicyn.

Subsiste l'accusation d'antisémitisme, récurrente et qui s'est à nouveau étalée dans le journal Le Monde en décembre 2018 lors de la célébration du centenaire de Solženicyn. Tempest cite George Steiner parlant de Solženicyn (« un génie pour le détail exact »), mais il faut bien voir que ce petit éloge contient du poison, et que George Steiner n'a cessé d'accuser Solženicyn pour son antisémitisme ${ }^{8}$. Ce qu'a fait également Šimon Markiš, fils du poète Perec Markiš, grand spécialiste d'Érasme. Markiš a longuement analysé la scène, où Parvus (Israël Gelfand), homme d'affaire allemand et haut responsable du Parti social-démocrate allemand, rend en songe une visite à Lenin, toujours en exil à Zürich, pour le persuader de prendre l'argent allemand et de passer en Russie avec l'aide de l'état-major de Guillaume II. On est là à un point brûlant (« le wagon plombé ») qui a soulevé des guerres historiennes himalayennes. Dans cette scène où Parvus apparaît sous la forme d'un hippopotame, Tempest, qui en a parlé avec Solženicyn, voit « l'apport de l'imagination pour mieux concentrer la réalité $»^{9}$. Expliquons : pour la logique de son récit, Soljenitsyne avait besoin de l'entrevue, mais il n'y en eu pas, ni à ce moment ni en ce lieu. La provoquer en songe était la solution. Cette étrange « solution »- le lecteur pressé ne remarque pas tout de suite que c'est un rêve -, ne saurait pourtant se ramener à un simple procédé narratif. Et d'ailleurs, aux commentateurs qui y ont vu un vestige d'antisémitisme médiéval, comme Šimon Markiš, Solženicyn a vivement répondu, sentant peut-être que son œuvre était attaquée sur un contrefort important ${ }^{10}$.

Enfin, reste un point qu'on ne saurait éviter et qui est assez déroutant : l'étrange omniprésence de Nietzsche dans cette étude. Nietzsche semble, à suivre le chapelet de citations qu'en fait Tempest, presque en accord avec Solženicyn : tous deux n’ont-ils pas annoncé le « crépuscule des idoles »? Mais Nietzsche est avant tout un samouraï de la pensée, un gladiateur - le fauve avec qui il lutte n'étant autre que lui-même - et il retourne chacune de ses pensées contre la précédente, comme un couteau dans une plaie. Or, Solženicyn, lui, est passé par les souffrances avant d'y voir clair, ce sont ces souffrances (guerre, goulag, cancer) qui lui ont dessillé les yeux, comme lui-même l'a confié à tant de reprises. Ce n'est pas l'aventure de la pensée qui l'a fait entrer dans l'Enfer, mais l'inverse. Et cette divergence de destin fondamentale dans les deux œuvres (comme les deux vies), est capitale. D'ailleurs l'une s'achève par dix années de folie, l'autre par une longue vieillesse, achèvement d'une vie-et-œuvre dans une unité miraculeuse. Un miracle né de la langue marmonnée dans la prison et couronné par cette gigantesque tentative de 
« domination du chaos » par l'écriture que Richard Tempest nous décrit. Quant à la victoire sur ce chaos, les doutes subsistent, mais toutes les épopées sont filles de la défaite.

$1-$ « A gigantic collage of texts and topoï ».

2 - « the human interest stickum that holds all these disparate pieces ».

3 - « The Soviet rule has its Homer in the case of Solzhenitsyn ».

4 - Gary poussa cette attitude de samouraï jusqu'à changer de nom et de style, duper tout le Paris littéraire, et gagner deux fois le Prix Goncourt.

5 - Andrej Nemzer. "Krasnoe koleso » Aleksandra Solženicyna, opyt pročtenija [La Roue rouge d'Aleksandr Solženicyn. Expérience de lecture], M., 2011.

6 - Olivier Clément, L'esprit de Soljénitsyne, P. : Stock, 1974.

7 - Aleksandr Sokurov est, au cinéma, celui qui a le plus magnifiquement montré le mystère de cette attente de la mort dans l'inaction guerrière.

8 - Le problème de l'antisémitisme de Solženicyn a rebondi avec un des derniers ouvrages, Deux siècles ensemble, qui est hors du champ de Tempest, puisque la fiction en est absente, mais qui comprend néanmoins des « chutes » de l'enquête de La Roue rouge. Le problème est vaste, les définitions sont toujours imprécises, et les passions toujours aux aguets. Solženicyn n'a évidemment rien d'un antisémite viscéral ; ce qui peut être discuté est sa définition de la notion de nation. Cf. Antoine Levy, "Alexandre Soljénitsyne, Deux siècles ensemble: 1795-1995. "Mais quand même..." ", Revue des études slaves, 75 ( 3-4), 2004, p. 519-531.

9 - « imagination as a means to concentrate reality ».

10 - Je précise que Markiš ne m'a pas convaincu, mais le connaisseur accompli d'Érasme qu'il était avait plus d'un argument.

\section{Georges Nivat}

Université de Genève 